were attributable to OCS, $31.3 \%(201,322)$ to screening in pregnancy (CSP), 30.6\% $(197,013)$ to diagnostic test (DT), and in $19.0 \%(122,347)$ the TR was not known. The number of OCStests increased by $114 \%$ in 2009, 15\% in 2010, and 7\% in 2011 compared to the previous year. Extrapolating, OCS covered $10 \%$ of the target population.

OCS PR was $5.3 \%$, higher than for other TR $(2.6 \%$ among CSP, and $3.9 \%$ among DT, p-value < 0.001). OCS PR was highest in women aged $15-19$ years $(7.1 \%)$ and $20-24(6.5 \%)$ compared to other age-groups ( $p$-value $<0.001)$.

Conclusion The proportion of young women testing positive for CT was high. OCS coverage in Germany is insufficient and did not increase substantially in the last years. OCS should be promoted among the target population and physicians. Awareness campaigns for young women should be implemented. Respective regulations should be expanded and remuneration to physicians for OCS offered.

\section{P3.029 SECULAR TRENDS OF CHLAMYDIA INFECTION AND TESTING: A CLOSE LOOK AT THE RISK FACTORS AND REGIONAL VARIATIONS OF A CANADIAN POPULATION, 1992-2008}

doi:10.1136/sextrans-2013-051184.0489

${ }^{1} \mathrm{~B}$ Yu, 'D Y Melesse, ${ }^{2} \mathrm{P}$ Caetano, 'S Leung, ${ }^{3} \mathrm{~B}$ Davies, ${ }^{4} \mathrm{~N}$ Low, ' $\mathrm{J}$ F Blanchard. 'University of Manitoba, Winnipeg, MB, Canada; ${ }^{2}$ Manitoba Health, Winnipeg, MB, Canada; ${ }^{3}$ Imperial College of London, London, UK; ${ }^{4}$ University of Bern, Bern, Switzerland

Background Screening and case-finding for Chlamydia infection among all sexually active men and women under the age of 25 , annually or during presentation to a health care provider were introduced in Canada and US in 2001. This strategy was aimed to reduce new Chlamydia infections and its long term adverse reproductive complications. The objective of this study is to explore the temporal trends of Chlamydia infections and test uptake in the targeted population of Manitoba.

Method We used Manitoba Cadham Provincial Laboratory (CPL) Chlamydia test data (1992 to 2008). CPL captured almost all sexually transmitted disease testing data of Manitoba. Descriptive statistics and logistic regression were used to describe the trend of Chlamydia infections and tests uptake over time.

Results Overall Chlamydia infection showed an initial flat trend in 1992-2001 and followed by a steady increasing in 2002-2008 trend in all women and men. Using Rural South as reference, the increasing infection rates are most prominent in Rural North rural, $\mathrm{OR}=6.12[\mathrm{CI}=6.06-6.17]$, Urban Core, $\mathrm{OR}=3.52$ [CI = 3.49-3.55], Urban non-Core, $\mathrm{OR}=3.06$ [3.04-3.08], Middle

\section{Abstract P3.029 Table 1}

\begin{tabular}{llll}
\hline \multicolumn{4}{l}{ Average annual crude rates (\% CI), 1992-2008 } \\
\hline Age/region & Infection & Positivity & Test Uptake \\
\hline Age $=<14$ & $0.02(0.02-0.03)$ & $13.3(9.49-17.14)$ & $0.18(0.16-0.19)$ \\
Age 15-19 & $1.58(1.48-1.69)$ & $11.07(10.93-12.33)$ & $14.32(14.90-15.50)$ \\
Age 20-24 & $1.77(1.75-1.96)$ & $7.04(6.62-7.45)$ & $25.19(24.78-25.59)$ \\
Age 25-29 & $0.83(0.76-0.90)$ & $3.88(3.54-4.22)$ & $22.02(21.64-22.39)$ \\
Age 30-34 & $0.34(0.30-0.39)$ & $2.28(1.98-2.58)$ & $15.28(14.98-15.28)$ \\
Age 35+ & $0.03(0.03-0.04)$ & $1.2(1.02-1.38)$ & $2.73(2.69-2.78)$ \\
Rural South & $1.21(1.13-1.29)$ & $9.44(8.79-10.10)$ & $13.42(13.14-13.70)$ \\
Urban Core & $0.57(0.53-0.62)$ & $6.4(5.91-6.90)$ & $9.36(9.18-9.53)$ \\
Urban Non-Core & $0.23(0.22-0.25)$ & $3.54(3.32-3.76)$ & $7.00(6.92-7.08)$ \\
Mid Rural & $0.26(0.23-0.28)$ & $5.52(4.97-6.08)$ & $4.86(4.74-4.97)$ \\
Rural South & $0.13(0.12-0.15)$ & $4.16(3.67-4.64)$ & $3.24(3.18-3.31)$ \\
\hline
\end{tabular}

Rural, OR $=1.78$ [1.76-1.79], $\mathrm{p}<0.0001)$. Chlamydia test uptake rates showed the same trend and prominent increased test uptakes since 2002 (annual increase $36 \%, O R=1.43$, CI $=1.42-1.44$, $\mathrm{p}<0.0001$ ), more in Rural North and Urban Core area in women aged 15-24 years. In younger women (aged 15-19) of Rural North, test positivity rates "outperformed" test uptake rates (annual increase $43 \%$ versus $22 \%$ ).

Conclusions The increasing trends of Chlamydia infection rates cannot be fully explained by the Chlamydia test uptakes in Manitoba young women. The geographic variations flag alarming signals for more effective core group targeted public health intervention.

\section{P3.030 A TARGETED WEB-BASED CHLAMYDIA TRACHOMATIS SCREENING STRATEGY FOR TESTING IN YOUNG PEOPLE AT RISK USING SOCIAL AND SEXUAL NETWORKS}

\author{
doi:10.1136/sextrans-2013-051184.0490
}

${ }^{1}$ K A T M Theunissen, ${ }^{1,2} \mathrm{C}$ J P A Hoebe, ${ }^{3} \mathrm{R}$ Crutzen, ${ }^{1,2} \mathrm{~A}$ Niekamp, ${ }^{4} \mathrm{C}$ Kara-Zaitri, ${ }^{3} \mathrm{~N}$ K de Vries, ${ }^{5,6,7} \mathrm{~J}$ E A M van Bergen, 5,8 M A B van der Sande, ${ }^{1,2} \mathrm{~N}$ H T M DukersMuijrers. 'Dept. of Sexual Health, Infectious Diseases and Environmental Health. Public Health Service South Limburg, Geleen, The Netherlands; '2Department of Medical Microbiology Maastricht Infection Centre (MINC), School for Public Health and Primary Care (CAPHRI), Maastricht University Medical Centre (MUMC+), Maastricht, The Netherlands; ${ }^{3}$ Department of Health Promotion, School for Public Health and Primary Care (CAPHRI), Maastricht University Medical Centre (MUMC+), Maastricht, The Netherlands; ${ }^{4}$ In-Fact, Bradford, UK; ${ }^{5}$ Centre for Infectious Disease Control, RIVM National Institute of Public Health and the Environment, Bilthoven, The Netherlands; ${ }^{6}$ The national institute for STI and AIDS Control, Amsterdam, The Netherlands, 'Department of General Practice, AMC-University of Amsterdam, Amsterdam, The Netherlands; ${ }^{8}$ Julius Center for Health Sciences and Primary Care, University Medical Center Utrecht, Utrecht, The Netherlands

Background Despite the availability of regular STI care by STI clinics and general practitioners many young people at high risk for Chlamydia trachomatis are hidden to care. Members of the social and sexual networks relating to Chlamydia positives typically show high risk, therefore these networks members are potentially valuable targets for interventions.

Methods Intervention Mapping (IM), a systematic approach to develop theory- and evidence-based interventions, was used to develop a strategy to target Ct testing towards young people who are currently hidden to care. Both clinical users (i.e. sexual health care nurses) and public users (i.e., young people) were closely involved in the IM process. A needs assessment study was carried out using semi-structured interviews among users $(\mathrm{n}=21)$, a literature search and by taking lessons learned from existing $\mathrm{Ct}$ screening programmes. Theoretical methods and practical applications to reach high risk young people and influence testing were selected and translated into specific programme components.

Results The IM approach resulted in the development of a secure and web-based outreach Chlamydia trachomatis screening strategy. $\mathrm{C} t$ testing and the motivation of peers were proposed as the desired behavioural outcomes. Key methods include web-based Respondent Driven Sampling (web-based RDS), starting from young Chlamydia positive sexual health care centre clients, to reach and motivate peers (i.e., sex partners and friends) to get tested. Furthermore, these sex partners and friends can also motivate peers in their networks to get tested, and so on.

Conclusions We believe this is a unique Chlamydia screening strategy that combines web-based RDS with the delivery of Chlamydia testing to high risk young people within their sexual and social networks. This approach can become an integral part in sexual health care for reaching high risk populations with Ct screening and treatment which is important for both the individual and public health level. 